\title{
Microbiome Study of Initial Gut Microbiota from Newborn Infants to Children Reveals that Diet Determines Its Compositional Development
}

\author{
Hye-Jin $\mathrm{Ku}^{\dagger}$, You-Tae Kim ${ }^{\dagger}$, and Ju-Hoon Lee* \\ Department of Food Science and Biotechnology, Graduate School of Biotechnology, Kyung Hee University, Yongin \\ 17104, Republic of Korea
}

\begin{abstract}
To understand the formation of initial gut microbiota, three initial fecal samples were collected from two groups of two breast milk-fed (BM1) and seven formula milk-fed (FM1) infants, and the compositional changes in gut microbiota were determined using metagenomics. Compositional change analysis during week one showed that Bifidobacterium increased from the first to the third fecal samples in the BM1 group (1.3\% to $35.1 \%)$, while Klebsiella and Serratia were detected in the third fecal sample of the FM1 group ( $4.4 \%$ and $34.2 \%$, respectively), suggesting the beneficial effect of breast milk intake. To further understand the compositional changes during progression from infancy to childhood (i.e., from three weeks to five years of age), additional fecal samples were collected from four groups of two breast milk-fed infants (BM2), one formula milk-fed toddler (FM2), three weaning food-fed toddlers (WF), and three solid food-fed children (SF). Subsequent compositional change analysis and principal coordinates analysis (PCOA) revealed that the composition of the gut microbiota changed from an infant-like composition to an adult-like one in conjunction with dietary changes. Interestingly, overall gut microbiota composition analyses during the period of progression from infancy to childhood suggested increasing complexity of gut microbiota as well as emergence of a new species of bacteria capable of digesting complex carbohydrates in WF and SF groups, substantiating that diet type is a key factor in determining the composition of gut microbiota. Consequently, this study may be useful as a guide to understanding the development of initial gut microbiota based on diet.
\end{abstract}

Keywords: Microbiome, initial gut microbiota, breast milk feeding, formula milk feeding, diet

Received: February 21, 2020 Accepted: April 3, 2020

First published online: April 9, 2020

* Corresponding author Phone: +82-31-201-3483 Fax: +82-31-204-8116 E-mail: juhlee@khu.ac.kr

${ }^{\dagger}$ These authors contributed equally to this work.

pISSN 1017-7825 elSSN 1738-8872

Copyright(C) 2020 by The Korean Society for Microbiology and Biotechnology
The gastrointestinal tract (GIT) of newborn infants is rapidly colonized by members of a variety of bacterial genera after birth $[1,2]$. It has been generally reported that the gut microbiota performs important functions for the human host, including protecting the infant's intestinal health by immune stimulation [3] and preventing infantile diarrhea [4]. Previous studies have suggested that the phylum Actinobacteria including Bifidobacterium is generally dominant in breast milk-fed infants, whereas the phylum Proteobacteria including Escherichia coli is dominant in formula-fed infants $[5,6]$, suggesting that human breast milk may influence the composition of gut microbiota in infants. However, little research into the formation and development of initial gut microbiota in infants has been conducted yet. In addition, the compositional conversion and progression from initial infantile gut microbiota to an adult-like one in children remain inadequately understood. Therefore, it is necessary to elucidate initial formation of gut microbiota in infants and the developmental mechanism of progression to one that is adult-like.

To characterize the composition and change of gut microbiota, fecal samples were obtained from newborn Korean infants who underwent a natural delivery and children up to five years old with an approval number (KHGIRB-19-192) from the Kyung Hee University Institutional Review Board (IRB). The total fecal DNA of each sample was extracted and purified following a previous protocol [7]. The 16S rRNA genes were PCR-amplified using a $926 \mathrm{~F} / 1505 \mathrm{R}$ primer set for GS-FLX+ pyrosequencing [7] or a $341 \mathrm{~F}$ and $805 \mathrm{R}$ primer set for Illumina MiSeq sequencing (Illumina, USA) [8]. The PCR products were sequenced using one of these next-generation sequencing methods. These $16 \mathrm{~S}$ rRNA sequencing reads were analyzed using the SILVA $16 \mathrm{~S}$ v128 database [9] for bacterial taxonomic assignments and the Microbial Genomics Module of the CLC Genomics Workbench software (Qiagen, Germany), following the default workflow and parameters for bacterial composition analysis.

For compositional analysis of initial gut microbiota in newborn infants, meconium and fecal samples were collected from two breast milk-fed (BM1) and seven formula milk-fed (FM1) infants (Table 1). Most of the 
Table 1. List of volunteers for collection of fecal samples.

\begin{tabular}{|c|c|c|c|c|c|c|}
\hline Subject & Sex & Age & Group $^{a}$ & Number of samples & Sample ID & Analysis method \\
\hline Newborn A & $\mathrm{F}$ & Newborn & BM1 & \multirow{6}{*}{$\begin{array}{l}\text { Three samples collected } \\
\text { at one week after birth }\end{array}$} & $\mathrm{A} 1 / \mathrm{A} 2 / \mathrm{A} 3$ & \multirow{9}{*}{$\begin{array}{l}454 \text { pyrosequencing } \\
(\text { GS FLX }+ \text { ) }\end{array}$} \\
\hline Newborn B & $\mathrm{F}$ & Newborn & BM1 & & $\mathrm{B} 1 / \mathrm{B} 2 / \mathrm{B} 3$ & \\
\hline Newborn C & $\mathrm{F}$ & Newborn & FM1 & & $\mathrm{C} 1 / \mathrm{C} 2 / \mathrm{C} 3$ & \\
\hline Newborn D & $\mathrm{F}$ & Newborn & FM1 & & $\mathrm{D} 1 / \mathrm{D} 2 / \mathrm{D} 3$ & \\
\hline Newborn E & M & Newborn & FM1 & & $\mathrm{E} 1 / \mathrm{E} 2 / \mathrm{E} 3$ & \\
\hline Newborn F & $\mathrm{F}$ & Newborn & FM1 & & $\mathrm{F} 1 / \mathrm{F} 2 / \mathrm{F} 3$ & \\
\hline Newborn G & M & Newborn & FM1 & \multirow{3}{*}{$\begin{array}{l}\text { Single sample collected } \\
\text { at one week after birth }\end{array}$} & G3 & \\
\hline Newborn $\mathrm{H}$ & M & Newborn & FM1 & & $\mathrm{H} 3$ & \\
\hline Newborn I & M & Newborn & FM1 & & $\mathrm{I} 3$ & \\
\hline Infant J & $\mathrm{F}$ & 3 weeks & BM2 & \multirow{9}{*}{$\begin{array}{l}\text { Three samples collected } \\
\text { at one-month intervals }\end{array}$} & $\mathrm{JE} 1 / 2 / 3$ & \multirow{9}{*}{$\begin{array}{l}\text { Illumina } \\
\text { (MiSeq) }\end{array}$} \\
\hline Infant K & $\mathrm{F}$ & 3 weeks & BM2 & & $J Y 1 / 2 / 3$ & \\
\hline Toddler L & M & 5 months & FM2 & & WJ1/2/3 & \\
\hline Toddler M & M & 6 months & $\mathrm{BM} 2 \rightarrow \mathrm{WF}$ & & $\mathrm{DH} 1 / 2 / 3^{\mathrm{b}}$ & \\
\hline Toddler N & $\mathrm{F}$ & 10 months & WF & & $\mathrm{MS} 1 / 2 / 3$ & \\
\hline Toddler O & M & 12 months & WF & & $\mathrm{RW} 1 / 2 / 3$ & \\
\hline Toddler P & $\mathrm{F}$ & 17 months & SF & & $\mathrm{EB} 1 / 2 / 3$ & \\
\hline Child Q & M & 3 years & $\mathrm{WF} \rightarrow \mathrm{SF}$ & & $\mathrm{JH}^{\mathrm{b}} / 2 / 3$ & \\
\hline Child R & $\mathrm{F}$ & 5 years & SF & & $\mathrm{MC} 1 / 2 / 3$ & \\
\hline
\end{tabular}

${ }^{a} \mathrm{BM} 1$, breast milk-fed infant (up to one week old); FM1, formula milk-fed infant (up to one week old); BM2, breast milk-fed infant (older than one week); FM2, formula milk-fed toddler (older than five months); WF, weaning food-fed toddler; SF, solid food-fed child.

${ }^{\mathrm{b}} \mathrm{DH} 3$ was sampled at the time at which the diet changed from BM2 to WF, while JH1 was sampled during WF feeding.

identified gut bacteria in BM1 and FM1 samples belong to four major phyla: Proteobacteria, Bacteroidetes, Firmicutes, and Actinobacteria (Fig. 1A). However, microbial succession was variable. In the BM1 group, Firmicutes (3.16\%-17.90\%) and Actinobacteria (1.09\%-31.19\%) increased but Bacteriodetes $(33.49 \%-1.41 \%)$ decreased (Fig. 1A). However, in the FM1 group, Proteobacteria $(5.90 \%-41.53 \%)$ increased but Firmicutes (51.99\%-26.58\%) decreased. At the genus level, this trend was supported by the observed increase in Bifidobacterium and Streptococcus but decrease in Bacteroides in the BM1 group and the observed increase in Serratia but decrease in Streptococcus in the FM1 group (Fig. 1B). This result suggests that the different microbial succession profiles between the BM1 and FM1 groups may be due to different feeding types. In particular, a rapid increase of Bifidobacterium in the BM1 group may be induced by human milk oligosaccharides (HMOs) in breast milk, suggesting that breastfeeding induces beneficial maturation of gut microbiota [10]. Human breast milk differs from cow milk as it contains sialylated and fucosylated oligosaccharides, which promote specific growth of Bifidobacterium [11, 12]. In addition, the FM1 group exhibited a more diverse microbiota including potential pathogens relative to the BM1 group, supporting the beneficial effect of breast milk. This increase in microbiota diversity of the FM1 group probably depends upon the contents of the formula milk.

A

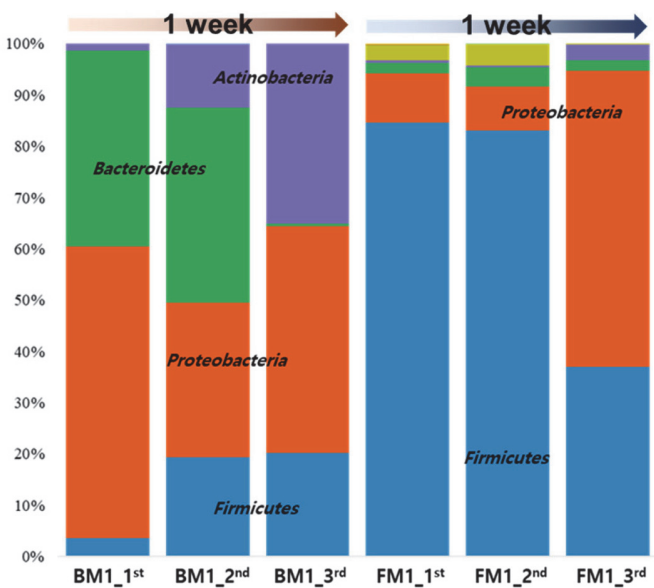

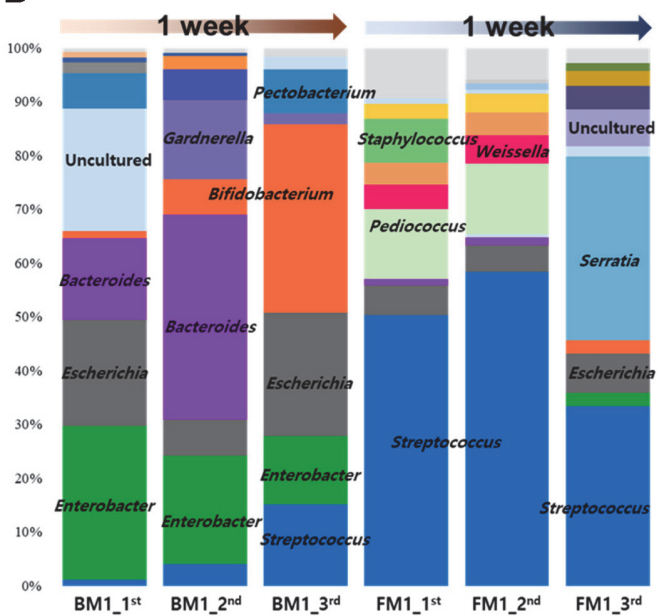

Fig. 1. Average compositional change in gut microbiota from birth to one week of age at the phylum (A) and genus levels (B). BM1, breast milk-fed infants; FM1, formula milk-fed infants. (BM1_1 ${ }^{\text {st }}$ contains A1 and B1; BM1_2 $2^{\text {nd }}$ contains A2 and B2; BM1_ $3^{\text {rd }}$ contains A3 and B3; FM1_1 $1^{\text {st }}$ contains C1, D1, E1, and F1; FM1_2 $2^{\text {nd }}$ contains C2, D2, E2, and F2; FM1_3 $3^{\text {rd }}$ contains $\mathrm{C} 3, \mathrm{D} 3, \mathrm{E} 3, \mathrm{~F} 3, \mathrm{G} 3, \mathrm{H} 3$, and I3). 
To further understand the compositional changes during development of infants to children (i.e., from three weeks to five years of age), 27 fecal samples were collected from four groups of two breast milk-fed infants (BM2), one formula milk-fed toddler (FM2), three weaning food-fed toddlers (WF), and three solid food-fed children (SF). At the phylum level, each group presents a unique major phyla pattern. In fecal samples of BM2 group, Firmicutes, Proteobacteria, and Actinobacteria were the major phyla identified, consistent with the similar composition of the $3^{\text {rd }}$ fecal samples in BM1 (Figs. 1A and 2A). However, the fecal samples of the FM2 group revealed a low composition of Actinobacteria, probably due to lack of HMOs in formula milk. This trend of reduced Actinobacteria was also observed in the WF group. According to increasing age of children, from BM2 to SF, Actinobacteria decreased but Firmicutes increased (Fig. 2A). In addition, Bacteroidetes became one of the

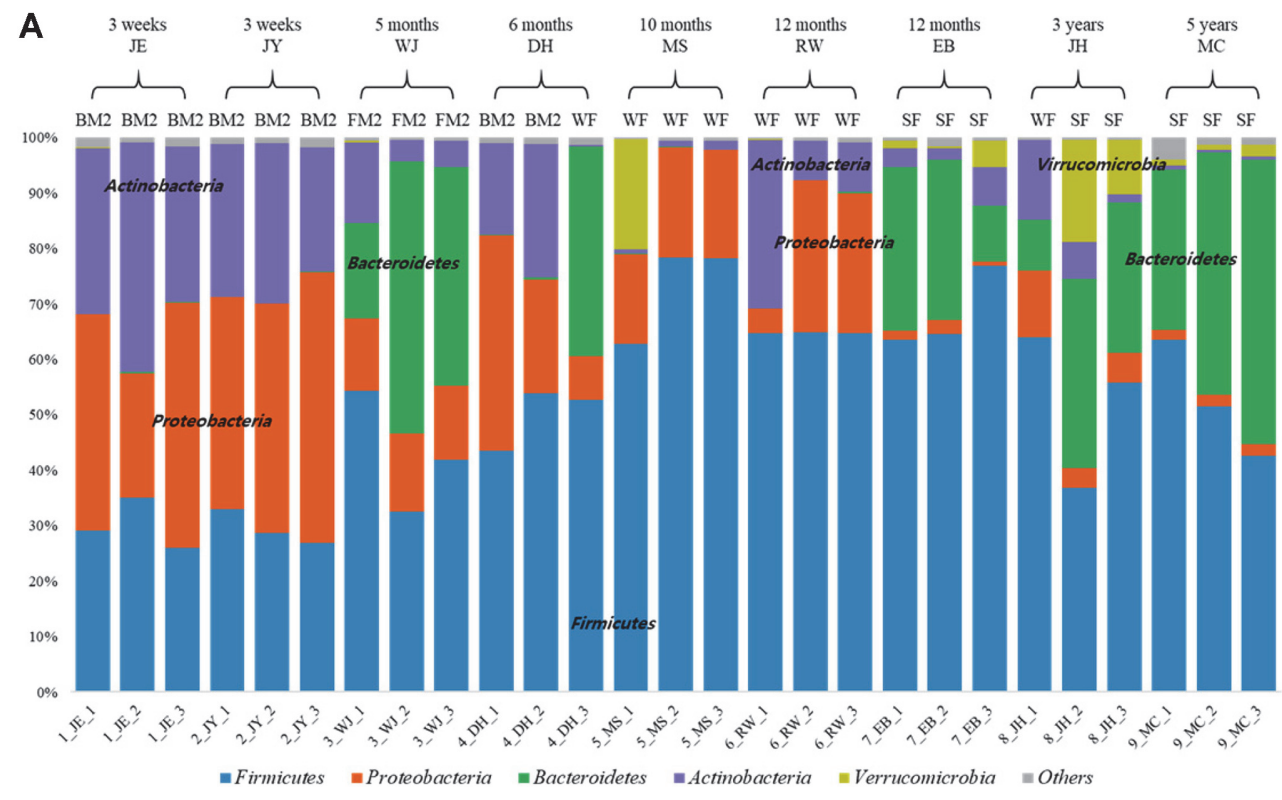

B

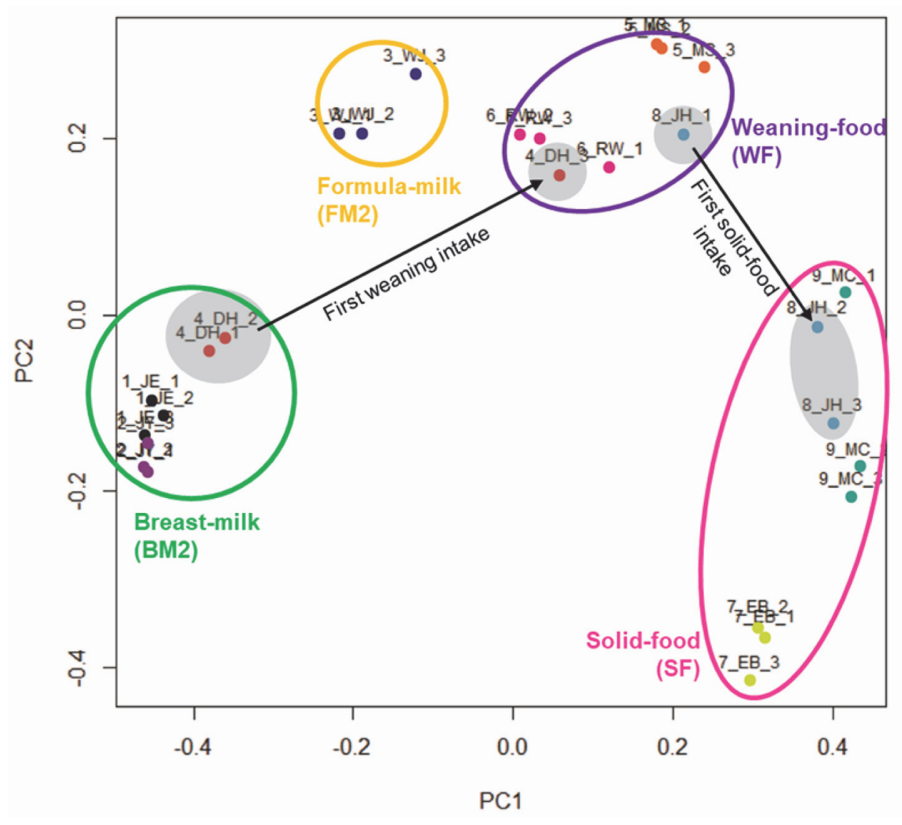

Fig. 2. Compositional change in gut microbiota from three weeks to five years of age for evaluating the impact of diet and individual variations. (A) The relative abundance of the taxonomic distributions in each individual sample. BM2, breast milk-fed infant (older than one week); FM2, formula milk-fed toddler (older than five months); WF, weaning food-fed toddler; SF, solid food-fed child. (B) PCoA (using Bray-Curtis matrix) plot from 27 fecal samples (obtained from nine donors). The figure represents sample ID and color code based on donor. Arrows indicate changes in gut microbiota according to dietary changes. 
major phyla in the SF group, suggesting that the composition of the gut microbiota in the SF group was altered from the initial infant gut microbiota to an adult-like one. Therefore, this feeding type may be responsible for the compositional changes in and progression of the gut microbiota from infancy to childhood. Subsequent PCoA analysis of these compositions of the gut microbiota showed that each group (i.e., BM2, FM2, WF, and SF) was located at a unique position relative to the others in the plot, supporting the above conjecture (Fig. 2B). Interestingly, the rapid dietary change of breast milk feeding (DH1 and DH2) to weaning-food feeding (DH3) in toddler M showed re-localization in the plot from the BM2 group to the WF group (Fig. 2B). Similarly, the rapid dietary change of weaning-food feeding ( $\mathrm{JH} 1)$ to solid-food feeding $(\mathrm{JH} 2$ and $\mathrm{JH} 3$ ) in child Q revealed relocalization from the WF group to the SF group, suggesting that only one dietary change could modulate the overall composition of the gut microbiota. Therefore, among the factors affecting gut microbiota in the growth stage, dietary changes may be the most significant factor.

To further understand the overall compositional changes in the gut microbiota during aging from infancy to five years of age, all compositional analysis results were merged. Then, the merged data were grouped according to feeding type as breast milk feeding (BM), formula-milk feeding (FM), weaning-food feeding (WF), and solid-

A

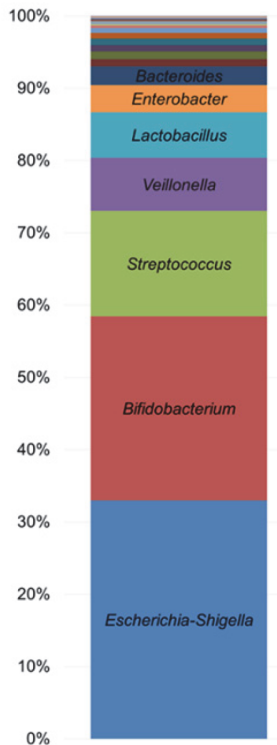

BM

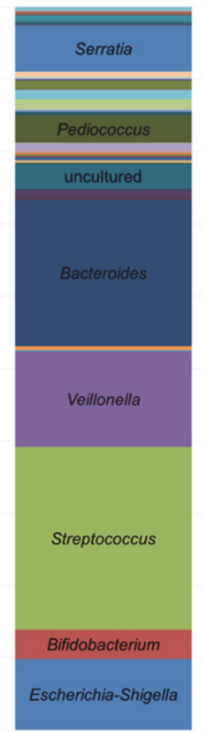

FM

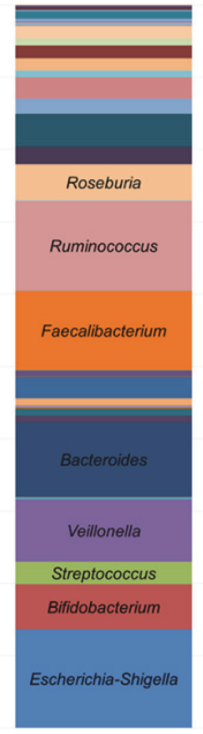

WF (Weaning-food)

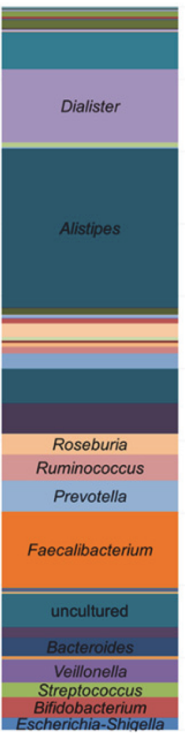

$\mathrm{SF}$

B

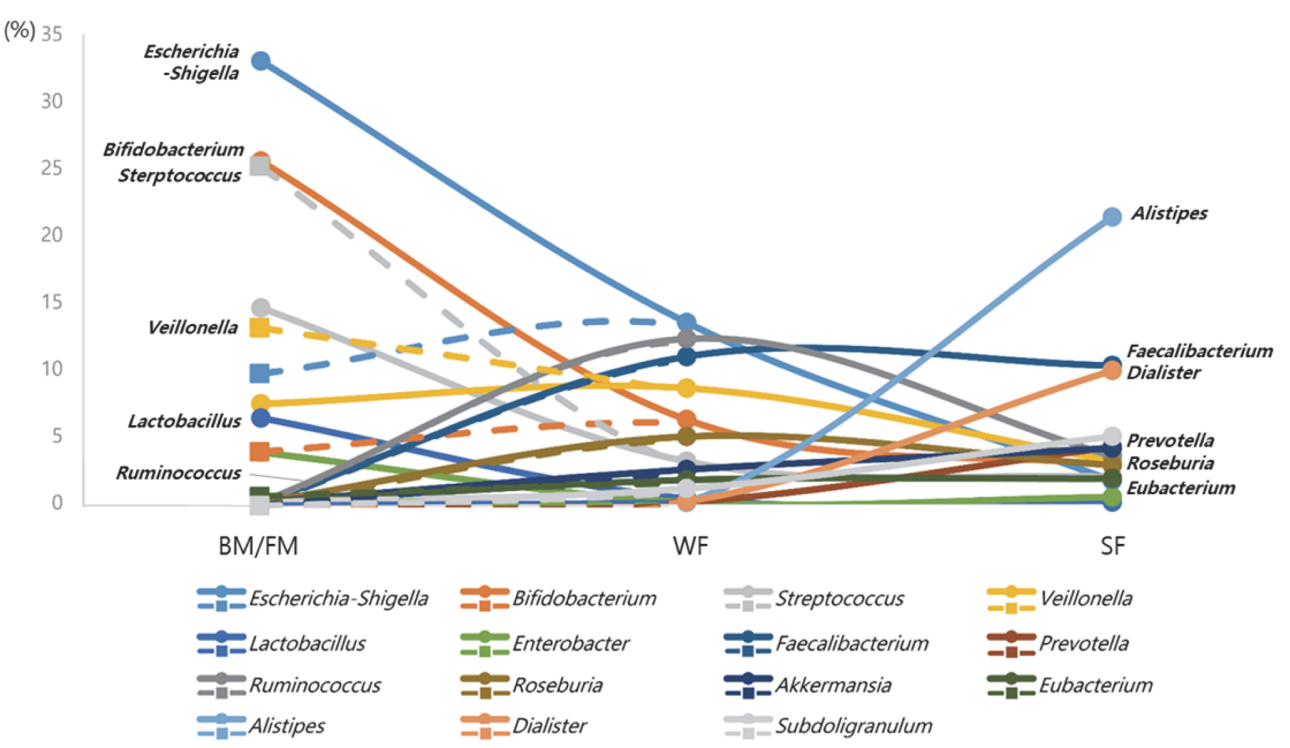

Fig. 3. Average compositional change in overall gut microbiota from infancy to childhood. (A) The relative abundance of each feeding type. (B) Compositional gut microbiota change in 15 major bacterial taxa by dietary change. Closed circle with a solid line, BM; closed square with dashed line, FM. 
food feeding (SF). Compared with the compositions of the gut microbiota in the BM and FM groups, the composition of gut microbiota became more complicated and new genera of bacteria including Alistipes, Dialister, Prevotella, Faecalibacterium, Ruminococcus, Roseburia, and Eubacterium were present in the WF and SF groups (Fig. 3A). To characterize the gut microbiota associated with dietary changes, the top 15 major bacterial taxa were selected, and their proportional changes were monitored (Fig. 3B). High proportions of Escherichia-Shigella and Bifidobacterium in BM and Streptococcus and Veillonella in FM gradually decreased in the WF group, finally reaching a proportion of less than $10 \%$ in the SF group. Increased proportions of Faecalibacterium, Ruminococcus, Roseburia, and Eubacterium were observed in the WF group. Finally, Prevotella, Akkermansia, Alistipes, and Dialister were colonized as major gut bacteria in the SF group (Fig. 3B). Therefore, it is possible that these newly emerged gut bacteria may be linked to intake of various nutrients as well as dietary fiber in children eating weaning or solid foods.

Previously, it was reported that solid food intake causes bile acid production by gut bacteria to regulate the balance of the gut microbiota [13]. In the SF group, a new genus of bile-tolerant bacterium, Alistipes, emerged and became one of the major gut bacteria, supporting this finding. Among the new bacteria, Dialister became a major gut bacterium, probably due to its ability to degrade host-indigestible complex carbohydrates or dietary fiber [14]. Other new bacteria such as Roseburia, Faecalibacterium, and Ruminococcus also show digestive abilities that survive under nondigestible dietary fiber-rich environments prompted by ingestion of solid foods and even produce butyrate as one of the major metabolites associated with regulation and balancing of gut microbiota [1416]. These newly emerged gut bacteria were observed in the healthy adult gut bacterial community, showing that maturation of the gut bacterial community to an adult-like configuration is facilitated by ingestion of solid foods [17].

Consequently, this study revealed the initiation and development of gut microbiota during progression from infancy to five years of age in Korean children. Therefore, it may provide a basis for a more comprehensive understanding of the maturation of the Korean initial gut microbiota and the role of the gut microbiota in lifelong health.

\section{Acknowledgments}

This work was supported by the Korea Institute of Planning and Evaluation for Technology in Food, Agriculture, and Forestry (IPET) through the Agricultural Microbiome R\&D Program (no. 918022-04-HD020) and the High Value-added Food Technology Development Program (no. 318090-03-HD040), funded by the Ministry of Agriculture, Food, and Rural Affairs (MAFRA).

\section{Conflict of Interest}

The authors have no financial conflicts of interest to declare.

\section{References}

1. Bezirtzoglou E. 1997. The intestinal microflora during the first weeks of life. Anaerobe. 3: 173-177.

2. Penders J, Thijs C, Vink C, Stelma FF, Snijders B, Kummeling I, et al. 2006. Factors influencing the composition of the intestinal microbiota in early infancy. Pediatrics 118: 511-521.

3. Hooper LV, Macpherson AJ. 2010. Immune adaptations that maintain homeostasis with the intestinal microbiota. Nat. Rev. Immunol. 10: 159-169.

4. Guarner F, Malagelada JR. 2003. Gut flora in health and disease. Lancet 361: 512-519.

5. Fan W, Huo G, Li X, Yang L, Duan C. 2014. Impact of diet in shaping gut microbiota revealed by a comparative study in infants during the first six months of life. J. Microbiol. Biotechnol. 24: 133-143.

6. Yoshioka H, Iseki K, Fujita K. 1983. Development and differences of intestinal flora in the neonatal period in breast-fed and bottlefed infants. Pediatrics 72: 317-321.

7. Ku H-J, Lee J-H. 2014. Development of a novel long-range $16 \mathrm{~S}$ rRNA universal primer set for metagenomic analysis of gastrointestinal microbiota in newborn infants. J. Microbiol. Biotechnol. 24: 812-822.

8. Herlemann DP, Labrenz M, Jürgens K, Bertilsson S, Waniek JJ, Andersson AF. 2011. Transitions in bacterial communities along the $2000 \mathrm{~km}$ salinity gradient of the Baltic Sea. ISME J. 5: 1571-1579.

9. Quast C, Pruesse E, Yilmaz P, Gerken J, Schweer T, Yarza P, et al. 2012. The SILVA ribosomal RNA gene database project: improved data processing and web-based tools. Nucleic Acids Res. 41: D590-D596.

10. Sela DA, Mills DA. 2010. Nursing our microbiota: molecular linkages between bifidobacteria and milk oligosaccharides. Trends Microbiol. 18: 298-307.

11. Coppa GV, Gabrielli O, Zampini L, Galeazzi T, Ficcadenti A, Padella L, et al. 2011. Oligosaccharides in 4 different milk groups, Bifidobacteria, and Ruminococcus obeum. J. Pediatr. Gastroenterol. Nutr. 53: 80-87.

12. Marcobal A, Sonnenburg J. 2012. Human milk oligosaccharide consumption by intestinal microbiota. Clin. Microbiol. Infect. 18: 12-15.

13. Jandhyala SM, Talukdar R, Subramanyam C, Vuyyuru H, Sasikala M, Reddy DN. 2015. Role of the normal gut microbiota. World J. Gastroenterol. 21: 8787-8803.

14. Koenig JE, Spor A, Scalfone N, Fricker AD, Stombaugh J, Knight R, et al. 2011. Succession of microbial consortia in the developing infant gut microbiome. Proc. Natl. Acad. Sci. USA 108 Suppl 1: 4578-4585.

15. Pryde SE, Duncan SH, Hold GL, Stewart CS, Flint HJ. 2002. The microbiology of butyrate formation in the human colon. FEMS Microbiol. Lett. 217: 133-139.

16. Roediger W. 1980. The colonic epithelium in ulcerative colitis: an energy-deficiency disease? Lancet. 316: 712-715.

17. Yatsunenko T, Rey FE, Manary MJ, Trehan I, Dominguez-Bello MG, Contreras M, et al. 2012. Human gut microbiome viewed across age and geography. Nature 486: 222-227. 